\title{
Band structure dynamics in indium wires
}

\author{
M. Chávez-Cervantes, ${ }^{*}$ R. Krause, S. Aeschlimann, and I. Gierz ${ }^{\dagger}$ \\ Max Planck Institute for the Structure and Dynamics of Matter, Center for Free Electron Laser Science, 22761 Hamburg, Germany
}

(Received 22 August 2017; revised manuscript received 19 April 2018; published 3 May 2018)

\begin{abstract}
One-dimensional indium wires grown on $\mathrm{Si}(111)$ substrates, which are metallic at high temperatures, become insulating below $\sim 100 \mathrm{~K}$ due to the formation of a charge density wave (CDW). The physics of this transition is not conventional and involves a multiband Peierls instability with strong interband coupling. This CDW ground state is readily destroyed with femtosecond laser pulses resulting in a light-induced insulator-to-metal phase transition. The current understanding of this transition remains incomplete, requiring measurements of the transient electronic structure to complement previous investigations of the lattice dynamics. Time- and angle-resolved photoemission spectroscopy with extreme ultraviolet radiation is applied to this end. We find that the transition from the insulating to the metallic band structure occurs within $\sim 660 \mathrm{fs}$, which is a fraction of the amplitude mode period. The long lifetime of the transient state $(>100 \mathrm{ps}$ ) is attributed to trapping in a metastable state in accordance with previous work.
\end{abstract}

DOI: 10.1103/PhysRevB.97.201401

Charge density wave (CDW) order [1] arises in lowdimensional solids due to the presence of large parallel sections on the Fermi surface separated by a nesting vector $q_{\mathrm{CDW}}$. A periodic rearrangement of the charges with a realspace periodicity of $2 \pi / q_{\mathrm{CDW}}$ opens up a band gap at the Fermi level resulting in a metal-to-insulator phase transition. This transition is driven by strong electron-phonon coupling (Peierls transition) and/or strong electronic correlations (Mott transition). It is typically discussed in terms of a complex order parameter, the amplitude of which represents the gap in the spectral function. Notably, CDW order (as well as other symmetry-broken ground states of solids) is readily destroyed with light, as quasiparticles are excited across this gap [2-8]. The observed time scales for CDW melting reveal important information about the microscopic physics of the CDW transition [9]. Time- and angle-resolved photoemission spectroscopy (tr-ARPES) allows for a momentum-, energy-, and time-resolved investigation of these phenomena, and highlights amplitude and phase oscillations of the order parameter, observed as periodic modulations of the size and the position of the band gap in momentum space, respectively [1,10-17].

Here we use tr-ARPES to investigate the light-induced insulator-to-metal transition in one-dimensional indium wires grown in situ on a $\mathrm{Si}(111)$ substrate. The structural dynamics of this transition have been revealed in previous time-resolved reflection high-energy electron diffraction (tr-RHEED) experiments $[18,19]$. Complementary measurements of the electronic structural dynamics are required for a complete picture of the light-induced phase transition.

At room temperature [Fig. 1(a)] the indium atoms form pairs of "zigzag" chains separated by one chain of silicon atoms, resulting in a $(4 \times 1)$ unit cell [20]. This crystal structure gives rise to three metallic bands [Fig. 1(c)] that cross the Fermi level at $k_{\mathrm{F}}=0.75 \AA^{-1}\left(m_{1}\right), k_{\mathrm{F}}=0.54 \AA^{-1}\left(m_{2}\right)$, and $k_{\mathrm{F}}=$

\footnotetext{
*mariana.chavez-cervantes@mpsd.mpg.de

†isabella.gierz@mpsd.mpg.de
}

$0.41 \AA^{-1}\left(m_{3}\right)$ corresponding to band fillings of $0.11\left(m_{1}\right)$, $0.38\left(m_{2}\right)$, and $0.50\left(m_{3}\right)$ [21,22]. The sketches in Figs. 1(c) and $1(\mathrm{~d})$ are based on density functional theory calculations from [23] and include photoemission matrix element effects that suppress the right branch of $m_{2}$ and the left branch of $m_{1}$ and $m_{3}$, respectively [24].

Below a critical temperature of about $100 \mathrm{~K}$ [Fig. 1(b)] a periodic lattice distortion drives the structure into an $(8 \times 2)$ phase, accompanied by a metal-to-insulator transition [21,25-27]. The dimerization of the outer indium atoms [red arrows in Fig. 1(b)] results in a doubling of the unit cell along the wires which gaps the half-filled band $m_{3}$. In addition, a shear distortion displaces neighboring indium chains along the direction parallel to the chains [green arrows in Fig. 1(b)] which transfers charge from $m_{1}$ to $m_{2}$. As a result, $m_{1}$ becomes unoccupied and $m_{2}$ almost half-filled. Due to the dimerization, $m_{2}$ also gaps. The size of the band gap below the Fermi level is $0.34 \mathrm{eV}$ for $m_{3}$ and ranges between 0.04 and $0.09 \mathrm{eV}$ depending on the momentum for $m_{2}[21,27]$. The insulating band structure is sketched in Fig. 1(d). The system possesses two amplitude modes, a shear mode at $0.6 \mathrm{THz}$ and a rotary mode which includes the dimerization of the outer indium atoms at $0.8 \mathrm{THz}$, that soften when approaching the critical temperature [28].

In Fig. 1(e) we show the potential energy surfaces for the $(4 \times 1)$ and $(8 \times 2)$ structures in orange and black, respectively, where $Q$ is the generalized reaction coordinate of the structural phase transition as defined in [18]. The potential energy surface for the $(8 \times 2)$ phase deviates from the standard mexican hat shape and exhibits an additional minimum at $Q=0$ that corresponds to a metastable $(4 \times 1)$ phase that is separated from the true $(8 \times 2)$ ground state by a potential barrier of 40 $\mathrm{meV}[18,28]$. Photoexcitation across the CDW gap is expected to bring the system from the black to the orange potential energy surface along the blue arrow. The expected tr-ARPES pump-probe signal for this transition is sketched in Fig. 1(f). This sketch is obtained by subtracting the $T<T_{\mathrm{C}}$ band structure [Fig. 1(d)] from the $T>T_{\mathrm{C}}$ band structure [Fig. 1(c)]. 

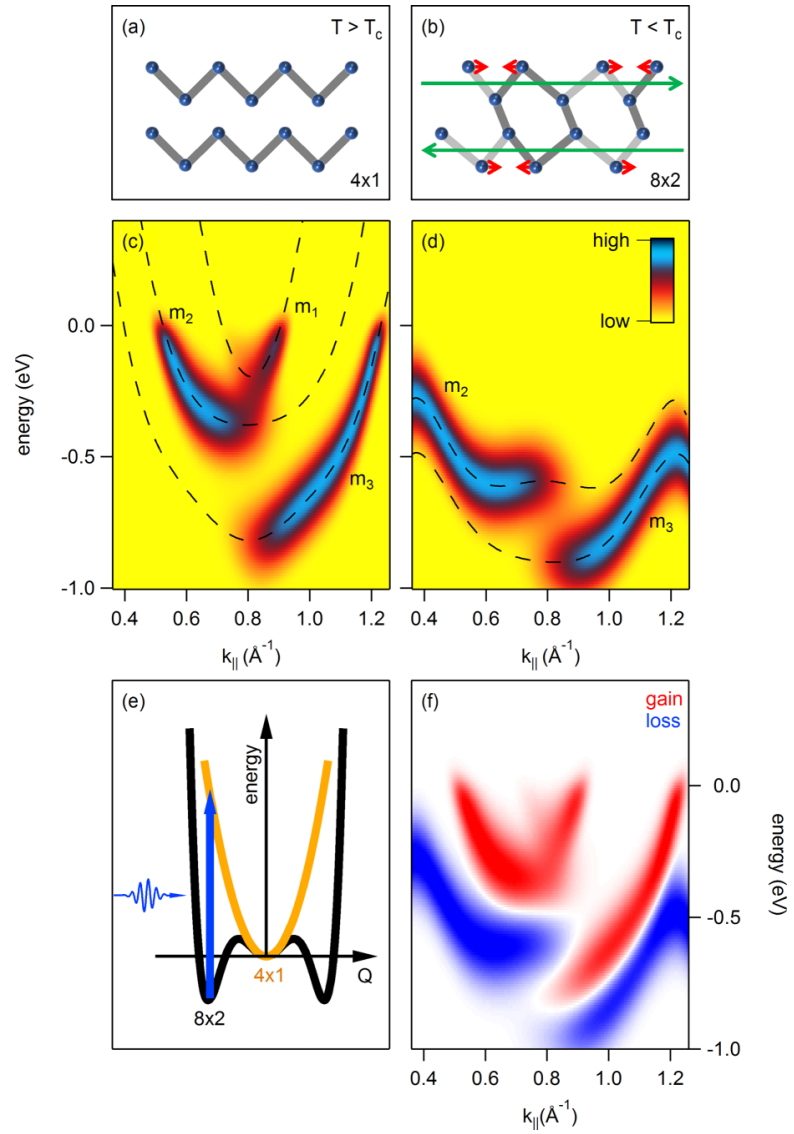

FIG. 1. Metal-to-insulator transition in one-dimensional indium wires on $\mathrm{Si}(111)$ : Sketch of the atomic structure above (a) and below (b) $T_{\mathrm{C}}$. Sketch of the electronic structure above (c) and below (d) $T_{\mathrm{C}}$ based on density functional theory calculations from [23]. (e) Sketch of the potential energy surface below (black) and above (orange) $T_{\mathrm{C}}$. $Q$ is the generalized reaction coordinate of the $(4 \times 1)$ to $(8 \times 2)$ transition as defined in [18]. The vertical blue arrow indicates the light-induced phase transition. (f) Difference between panels (c) and (d) simulating the pump-probe signal for the light-induced phase transition.
Details about the sample preparation and the tr-ARPES setup are given in the Supplemental Material [29].

Figure 2 shows the equilibrium band structure at $T=40$ $\mathrm{K}$ [Fig. 2(a)] together with the response of the band structure to photoexcitation of the system with femtosecond pulses at $1.0 \mathrm{eV}$ photon energy with a fluence of $5.6 \mathrm{~mJ} / \mathrm{cm}^{2}$ [Fig. 2(b)]. At the peak of the electronic excitation $(t=0.1 \mathrm{ps})$ we observe a broad distribution of photoexcited electrons and holes. After $1.1 \mathrm{ps}$, the pump-probe signal is mainly confined to the area below the Fermi level and closely resembles the sketch in Fig. 1(f) for a light-induced insulator-to-metal phase transition. We observe no further relaxation within $3 \mathrm{ps}$, indicating a long-lived transient state.

In order to quantify the CDW melting and relaxation dynamics we integrate the pump-probe signal over the areas indicated by the black boxes in Fig. 2(b) yielding the pumpprobe traces in Fig. 3(a). From exponential fits to the data [thick lines in Fig. 3(a); for details, see [29]] we determine the rise and decay times shown in Figs. 3(b) and 3(c), respectively. We attribute the short-lived pump-probe signal at high energies to the electronic excitation. The long-lived response ( $>100 \mathrm{ps})$ with the slow rise time $(660 \pm 180 \mathrm{fs})$ below the Fermi level reflects the melting of the CDW: The band gaps in $m_{2}$ and $m_{3}$ close and $m_{1}$ shifts back below the Fermi level.

For comparison, we present tr-ARPES data taken at room temperature in the $(4 \times 1)$ phase in Fig. 4. Figures 4(a), 4(b) and 4(c) show the equilibrium photocurrent, the simulated pumpprobe signal assuming a hot electronic distribution for the transient state, and the measured pump-probe signal for pumpprobe time delays of 0.1 and 1.0 ps for a pump photon energy of $1.55 \mathrm{eV}$ and a pump fluence of $2 \mathrm{~mJ} / \mathrm{cm}^{2}$, respectively. We confirmed that the difference in pump photon energy and fluence with respect to the data taken at $40 \mathrm{~K}$ does not affect the conclusions drawn in this section [29]. In contrast to the data for the $(8 \times 2)$ phase in Figs. 2 and 3, the pump-probe signal for the $(4 \times 1)$ phase is almost gone after $1 \mathrm{ps}$. Individual pump-probe traces taken at different energies [Fig. 4(d)] show only a small increase of the rise (decay) time from $130 \pm 5 \mathrm{fs}(590 \pm 10 \mathrm{fs})$ to $240 \pm 20 \mathrm{fs}(1.10 \pm 0.03 \mathrm{ps})$ when approaching the Fermi
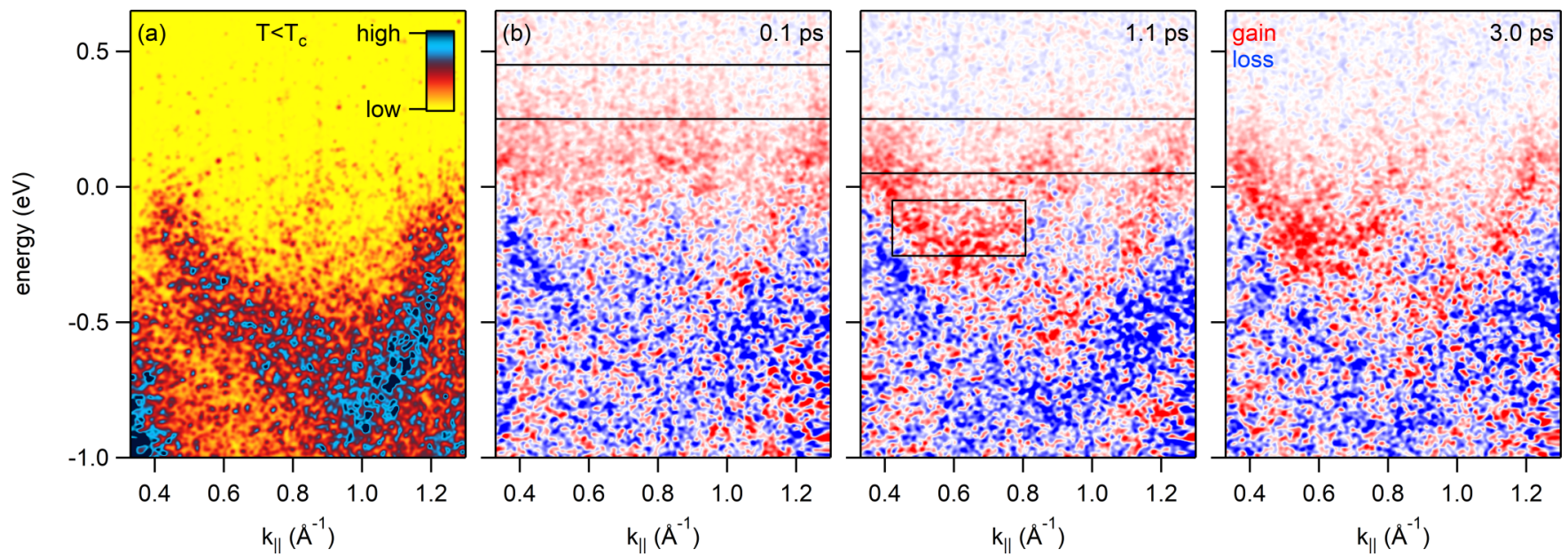

FIG. 2. Light-induced insulator-to-metal transition viewed by tr-ARPES at $T=40 \mathrm{~K}$. (a) Equilibrium photocurrent. (b) Pump-induced changes of the photocurrent at $t=0.1 \mathrm{ps}, t=1.1 \mathrm{ps}$, and $t=3.0 \mathrm{ps}$ for a pump photon energy of $1.0 \mathrm{eV}$ and a pump fluence of $5.6 \mathrm{~mJ} / \mathrm{cm}^{2}$. The black boxes (centered at \pm 0.15 and $+0.35 \mathrm{eV}$ ) indicate the area of integration for the pump-probe traces in Fig. 3(a). 

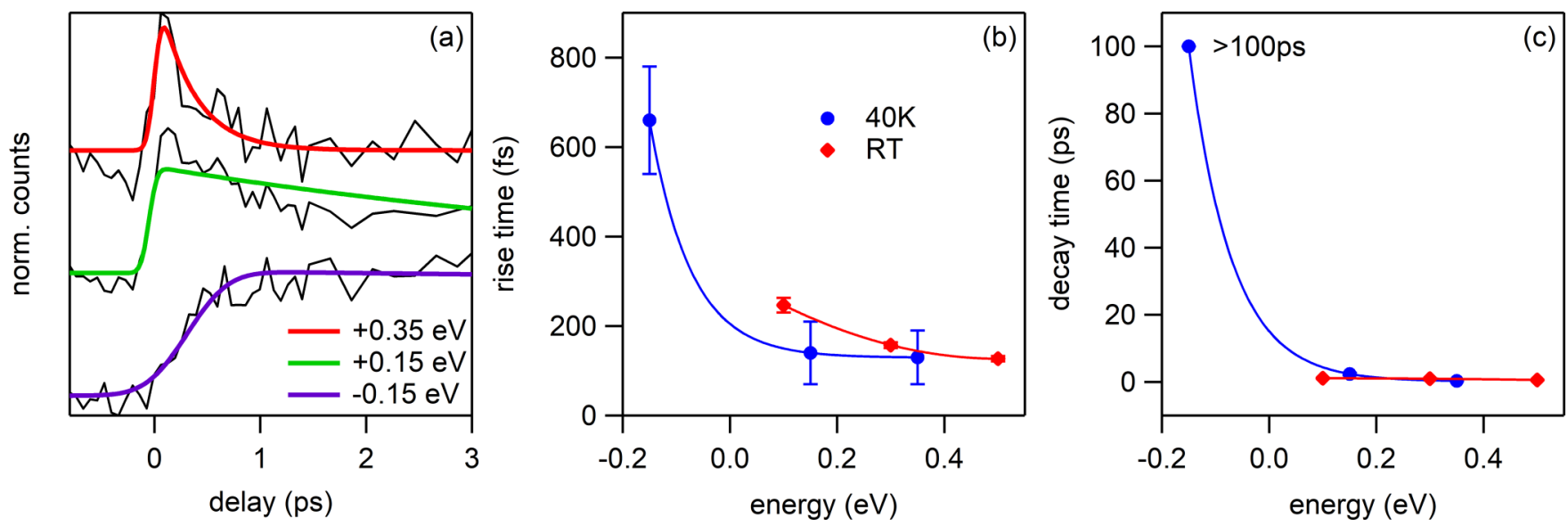

FIG. 3. Energy dependence of the pump-probe signal at $T=40 \mathrm{~K}$. (a) Pump-probe traces (thin lines) obtained by integrating the pumpinduced changes of the photocurrent over the areas indicated by black boxes in Fig. 2(b). Thick lines are exponential fits. (b) Rise and (c) decay time as a function of energy. The values for the room temperature (RT) measurement from Fig. 4 are included in (b) and (c) for direct comparison. Continuous lines in (b) and (c) are guides to the eye.
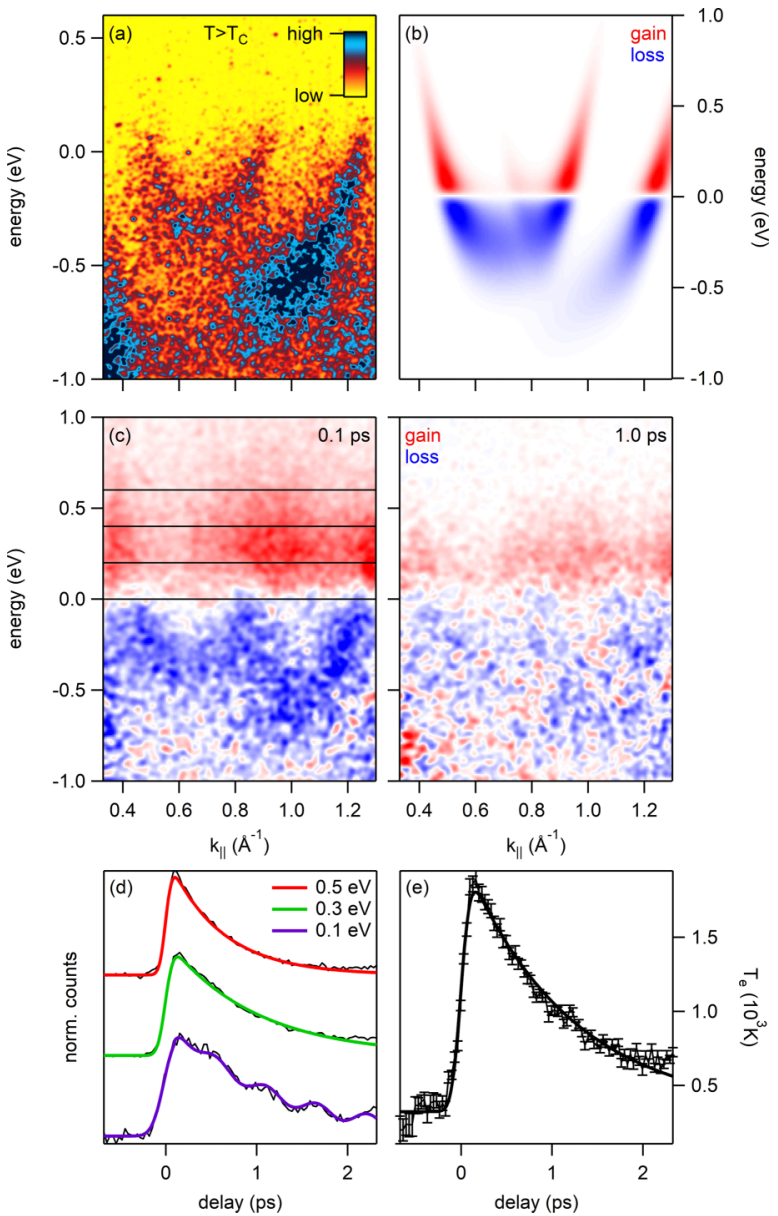

FIG. 4. Dynamics at room temperature. (a) Equilibrium photocurrent. (b) Simulated pump-probe signal assuming a hot electronic distribution for the transient state. (c) Pump-induced changes of the photocurrent at $t=0.1 \mathrm{ps}$ and $t=1.1 \mathrm{ps}$ for a pump photon energy of $1.55 \mathrm{eV}$ and a pump fluence of $2 \mathrm{~mJ} / \mathrm{cm}^{2}$. The black boxes indicate the area of integration for the pump-probe traces in panel (d). Thick lines in (d) are exponential fits. The pump-probe trace at $0.1 \mathrm{eV}$ shows coherent oscillations at $1.8 \pm 0.1 \mathrm{THz}$. (e) Electronic temperature as a function of pump-probe delay together with exponential fit. level [see red data points in Figs. 3(b) and 3(c)]. The close resemblance between Figs. 4(b) and 4(c) indicates that the pump-probe signal can be understood in terms of a hot electronic distribution characterized by the time-dependent electronic temperature shown in Fig. 4(e) (for details, see [29]) without any underlying changes of the band structure. The cooling time for the hot electrons is found to be $1.18 \pm 0.05 \mathrm{ps}$ from exponential fitting.

Next, we compare our results to previous tr-RHEED measurements of the lattice dynamics [18,19]. In Ref. [19], the $(8 \times 2)$ to $(4 \times 1)$ transition was found to occur within $350 \mathrm{fs}$ ( $680 \mathrm{fs}$ according to our definition of the rise time; see [29]) in excellent agreement with our own value of $660 \pm 120 \mathrm{fs}$. This number is slightly longer than $1 / 4$ period of the two amplitude modes (310 and $420 \mathrm{fs}$, respectively [28]) which set the lower limit for CDW melting in a Peierls picture. The recovery time of the $(8 \times 2)$ phase was found to be $440 \mathrm{ps}$ with tr-RHEED [18], consistent with our value of $>100 \mathrm{ps}$ for the recovery of the insulating band structure.

The long lifetime of the transient $(4 \times 1)$ phase in the tr-RHEED experiments was attributed to trapping in the metastable $(4 \times 1)$ phase shown in Fig. 1(e) [18]. Relaxation into the $(8 \times 2)$ phase was proposed to happen via condensation nuclei that grow with a velocity of $850 \mathrm{~m} / \mathrm{s}$ [18].

In agreement with Ref. [19] we find no indications for coherent amplitude mode oscillations in our data below $T_{\mathrm{C}}$. This has been attributed to strong damping of the amplitude modes due to rapid energy transfer to various phonon modes of the surface and the substrate [19]. However, we observe coherent oscillations in the pump-probe trace centered at $+0.1 \mathrm{eV}$ at room temperature in Fig. 4(d). The frequency of this oscillation, determined from a sinusoidal fit, is $1.8 \pm 0.1$ THz. Previous Raman measurements show a peak at the same frequency that was attributed to a phonon that periodically modulates the distance between two neighboring indium chains [30,31].

In summary, we have shown that the transition from the insulating to the metallic band structure in one-dimensional indium wires on $\mathrm{Si}(111)$ occurs on a timescale slightly longer than $1 / 4$ period of the amplitude modes of the system 
consistent with a Peierls transition. After photoexcitation the system gets trapped in a metastable $(4 \times 1)$ phase with $>100$ ps lifetime. Our findings are in excellent agreement with previous complementary investigations of the lattice dynamics with tr-RHEED [18,19].
We thank H. Bromberger for technical support and A. Cavalleri for careful reading of the manuscript and many valuable comments. This work received financial support from the German Science Foundation via the SFB 925 "Light induced dynamics and control of correlated quantum systems".
[1] G. Grüner, Density Waves in Solids (Perseus Publishing, Cambridge, MA, 1994).

[2] A. Cavalleri, C. Tóth, C. W. Siders, J. A. Squier, F. Ráksi, P. Forget, and J. C. Kieffer, Phys. Rev. Lett. 87, 237401 (2001).

[3] S. Iwai, M. Ono, A. Maeda, H. Matsuzaki, H. Kishida, H. Okamoto, and Y. Tokura, Phys. Rev. Lett. 91, 057401 (2003).

[4] A. Cavalleri, T. Dekorsy, H. H. W. Chong, J. C. Kieffer, and R. W. Schoenlein, Phys. Rev. B 70, 161102(R) (2004).

[5] M. Chollet, L. Guerin, N. Uchida, S. Fukaya, H. Shimoda, T. Ishikawa, K. Matsuda, T. Hasegawa, A. Ota, H. Yamochi, G. Saito, R. Tazaki, S. Adachi, and S. Koshihara, Science 307, 86 (2005).

[6] A. Tomeljak, H. Schäfer, D. Städter, M. Beyer, K. Biljakovic, and J. Demsar, Phys. Rev. Lett. 102, 066404 (2009).

[7] M. Eichberger, H. Schäfer, M. Krumova, M. Beyer, J. Demsar, H. Berger, G. Moriena, G. Sciaini, and R. J. D. Miller, Nature (London) 468, 799 (2010).

[8] S. Hellmann, M. Beye, C. Sohrt, T. Rohwer, F. Sorgenfrei, H. Redlin, M. Kalläne, M. Marczynski-Bühlow, F. Hennies, M. Bauer, A. Föhlisch, L. Kipp, W. Wurth, and K. Rossnagel, Phys. Rev. Lett. 105, 187401 (2010).

[9] C. Sohrt, A. Stange, M. Bauer, and K. Rossnagel, Faraday Discuss. 171, 243 (2014).

[10] L. Perfetti, P. A. Loukakos, M. Lisowski, U. Bovensiepen, H. Berger, S. Biermann, P. S. Cornaglia, A. Georges, and M. Wolf, Phys. Rev. Lett. 97, 067402 (2006).

[11] F. Schmitt, P. S. Kirchmann, U. Bovensiepen, R. G. Moore, L. Rettig, M. Krenz, J.-H. Chu, N. Ru, L. Perfetti, D. H. Lu, M. Wolf, I. R. Fisher, and Z.-X. Shen, Science 321, 1649 (2008).

[12] T. Rohwer, S. Hellmann, M. Wiesenmayer, C. Sohrt, A. Stange, B. Slomski, A. Carr, Y. Liu, L. M. Avila, M. Kalläne, S. Mathias, L. Kipp, K. Rossnagel, and M. Bauer, Nature (London) 471, 490 (2011).

[13] J. C. Petersen, S. Kaiser, N. Dean, A. Simoncig, H. Y. Liu, A. L. Cavalieri, C. Cacho, I. C. E. Turcu, E. Springate, F. Frassetto, L. Poletto, S. S. Dhesi, H. Berger, and A. Cavalleri, Phys. Rev. Lett. 107, 177402 (2011).

[14] S. Hellmann, T. Rohwer, M. Kalläne, K. Hanff, C. Sohrt, A. Stange, A. Carr, M. M. Murnane, H. C. Kapteyn, L. Kipp, M. Bauer, and K. Rossnagel, Nat. Commun. 3, 1069 (2012).

[15] H. Y. Liu, I. Gierz, J. C. Petersen, S. Kaiser, A. Simoncig, A. L. Cavalieri, C. Cacho, I. C. E. Turcu, E. Springate, F. Frassetto, L. Poletto, S. S. Dhesi, Z.-A. Xu, T. Cuk, R. Merlin, and A. Cavalleri, Phys. Rev. B 88, 045104 (2013).

[16] S. Mathias, S. Eich, J. Urbancic, S. Michael, A. V. Carr, S. Emmerich, A. Stange, T. Popmintchev, T. Rohwer, M. Wiesenmayer, A. Ruffing, S. Jakobs, S. Hellmann, P. Matyba, C. Chen, L. Kipp, M. Bauer, H. C. Kapteyn, H. C. Schneider,
K. Rossnagel, M. M. Murnane, and M. Aeschlimann, Nat. Commun. 7, 12902 (2015).

[17] L. Rettig, R. Cortés, J.-H. Chu, I. R. Fisher, F. Schmitt, R. G. Moore, Z.-X. Shen, P. S. Kirchmann, M. Wolf, and U. Bovensiepen, Nat. Commun. 7, 10459 (2015).

[18] S. Wall, B. Krenzer, S. Wippermann, S. Sanna, F. Klasing, A. Hanisch-Blicharski, M. Kammler, W. G. Schmidt, and M. Hornvon Hoegen, Phys. Rev. Lett. 109, 186101 (2012).

[19] T. Frigge, B. Hafke, T. Witte, B. Krenzer, C. Streubühr, A. Samad Syed, V. Mikšić Trontl, I. Avigo, P. Zhou, M. Ligges, D. von der Linde, U. Bovensiepen, M. Horn-von Hoegen, S. Wippermann, A. Lüke, S. Sanna, U. Gerstmann, and W. G. Schmidt, Nature (London) 544, 207 (2017).

[20] O. Bunk, G. Falkenberg, J. H. Zeysing, L. Lottermoser, R. L. Johnson, M. Nielsen, F. Berg-Rasmussen, J. Baker, and R. Feidenhans'l, Phys. Rev. B 59, 12228 (1999).

[21] J. R. Ahn, J. H. Byun, H. Koh, E. Rotenberg, S. D. Kevan, and H. W. Yeom, Phys. Rev. Lett. 93, 106401 (2004).

[22] T. Abukawa, M. Sasaki, F. Hisamatsu, T. Goto, T. Kinoshita, A. Kakizaki, and S. Kono, Surf. Sci. 325, 33 (1995).

[23] S.-W. Kim and J.-H. Cho, Phys. Rev. B 93, 241408(R) (2016).

[24] H. Morikawa, C. C. Hwang, and H. W. Yeom, Phys. Rev. B 81, 075401 (2010).

[25] H. W. Yeom, S. Takeda, E. Rotenberg, I. Matsuda, K. Horikoshi, J. Schaefer, C. M. Lee, S. D. Kevan, T. Ohta, T. Nagao, and S. Hasegawa, Phys. Rev. Lett. 82, 4898 (1999).

[26] C. González, J. Ortega, and F. Flores, New J. Phys. 7, 100 (2005).

[27] Y. J. Sun, S. Agario, S. Souma, K. Sugawara, Y. Tago, T. Sato, and T. Takahashi, Phys. Rev. B 77, 125115 (2008).

[28] E. Jeckelmann, S. Sanna, W. G. Schmidt, E. Speiser, and N. Esser, Phys. Rev. B 93, 241407(R) (2016).

[29] See Supplemental Material at http://link.aps.org/supplemental/ 10.1103/PhysRevB.97.201401 for further information about sample growth, tr-ARPES setup, and data analysis, including Refs. [22,32-35].

[30] K. Fleischer, S. Chandola, N. Esser, W. Richter, and J. F. McGilp, Phys. Rev. B 76, 205406 (2007).

[31] E. Speiser, N. Esser, S. Wippermann, and W. G. Schmidt, Phys. Rev. B 94, 075417 (2016).

[32] J. L. Stevens, M. S. Worthington, and I. S. T. Tsong, Phys. Rev. B 47, 1453 (1993).

[33] K. Sakamoto, H. Ashima, H. W. Yeom, and W. Uchida, Phys. Rev. B 62, 9923 (2000).

[34] J.-L. Lin, D. Y. Petrovykh, J. Viernow, F. K. Men, D. J. Seo, and F. J. Himpsel, J. Appl. Phys. 84, 255 (1998).

[35] F. Frassetto, C. Cacho, C. A. Froud, I. C. E. Turcu, P. Villoresi, W. A. Bryan, E. Springate, and L. Poletto, Opt. Express 19, 19169 (2011). 